\title{
Borrelia burgdorferi Sensu Stricto DNA in Field-Collected Haemaphysalis longicornis Ticks, Pennsylvania, United States
}

\author{
Keith J. Price, Christine B. Graham, Bryn J. Witmier, Holly A. Chapman, Brooke L. Coder, \\ Christian N. Boyer, Erik Foster, Sarah E. Maes, Ying Bai, Rebecca J. Eisen, and Andrew D. Kyle
}

We collected questing Haemaphysalis longicornis ticks from southeastern counties of Pennsylvania, USA. Of 263 ticks tested by PCR for pathogens, 1 adult female was positive for Borrelia burgdorferi sensu stricto, yielding a $0.4 \%$ infection rate. Continued monitoring of this invasive tick is essential to determine its public health role.

Rorrelia burgdorferi sensu stricto is the causative

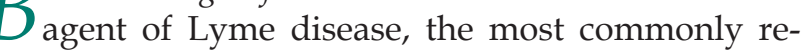
ported vectorborne disease in North America (1). In Pennsylvania, which is first in the United States in the number of reported Lyme disease cases, the spirochete has been identified in nearly $50 \%$ of adult Ixodes scapularis ticks, the primary vector (2). In 2018, Pennsylvania initiated a statewide active surveillance program to monitor tick distribution and density, by county, and tickborne pathogen prevalence. Although focused primarily on collecting and testing Ixodes scapularis ticks, initial surveillance efforts recovered, among other species, Haemaphysalis longicornis (Asian longhorned tick), an exotic species recently detected in North America (3), providing quantitative records of their presence in Pennsylvania public lands (4).

Since its US discovery in New Jersey during 2017, the number of states that have detected $H$. longicor$n i s$ ticks has increased rapidly. In its native range, $H$. longicornis ticks have been found to carry a variety of pathogens endemic to Pennsylvania, including $B$. burgdorferi (5). However, because the ecologic characteristics and the pathogen diversity and prevalence of

Author affiliations: Pennsylvania Department of Environmental Protection, Harrisburg, Pennsylvania, USA (K.J. Price, B.J. Witmier, H.A. Chapman, B.L. Coder, C.N. Boyer, A.D. Kyle); Centers for Disease Control and Prevention, Fort Collins, Colorado, USA (C.B. Graham, E. Foster, S.E. Maes, Y. Bai, R.J. Eisen)

DOI: https://doi.org/10.3201/eid2702.201552
H. longicornis ticks in the United States are understudied, potential epidemiologic risks there remain unknown. We report surveillance program data on the presence of pathogen-infected H. longicornis in public areas in Pennsylvania.

\section{The Study}

We performed surveillance activities weekly in 38 Pennsylvania counties during May 1-September 6, 2019, capturing peak nymphal I. scapularis ticks, in addition to adult and nymphal $H$. longicornis tick densities (6). Sampling sites, primarily high-use public areas in deciduous forests, were selected for high risk of recreational and occupational tick encounters and suitable I. scapularis and reported H. longicornis tick habitat (6).

Collection processes were standardized to minimize spatial and temporal bias. We collected questing ticks by dragging a $1 \mathrm{~m}^{2}$ white felt cloth over vegetation and leaf litter for 100-600 m. We examined cloths every $10 \mathrm{~m}$ and transferred recovered ticks into vials containing $80 \%$ ethanol, which we shipped to a central laboratory where they were stored at $-80^{\circ} \mathrm{C}$ until being identified using morphological keys.

We tested the majority $(84 \%)$ of collected $H$. longicornis nymphs and adults for pathogens, then retained the rest as voucher specimens. We prepared DNA extracts from individual $H$. longicornis tick homogenates on the KingFisher Flex Purification System with the MagMAX CORE Nucleic Acid Purification Kit (ThermoFisher Scientific, https:// www.thermofisher.com). We tested each extract for B. burgdorferi sensu stricto, B. mayonii, B. miyamotoi, and Babesia microti using probe-based real-time PCR assays comprising multiple targets for each pathogen (Table). We amplified a segment of the Borrelia dipeptidyl aminopeptidase (PepX) gene using 
B. burgdorferi DNA in Haemaphysalis longicornis Ticks

Table. Pathogen targets included in real-time PCR testing of individual Haemaphysalis longicornis ticks, Pennsylvania, USA * $\dagger$

\begin{tabular}{|c|c|c|c|c|c|}
\hline \multirow[b]{2}{*}{ PCR target } & \multicolumn{4}{|c|}{ Pathogen } & \multirow[b]{2}{*}{ Reference } \\
\hline & $\begin{array}{l}\text { Borrelia burgdorferi } \\
\text { sensu stricto }\end{array}$ & B. mayonii & B. miyamotoi & Babesia microti & \\
\hline Borrelia 16S rDNA & $\ddagger$ & $\ddagger$ & $\ddagger$ & NA & $(7)$ \\
\hline B. burgdorferi sensu lato fliD & $\ddagger$ & $\ddagger$ & NA & NA & (8) \\
\hline B. burgdorferi sensu stricto oppA2 & $\ddagger$ & NA & NA & NA & (9) \\
\hline B. mayonii oppA2 & NA & $\ddagger$ & NA & NA & (9) \\
\hline Borrelia miyamotoi purB & NA & NA & $\ddagger$ & NA & (9) \\
\hline B. miyamotoi glpQ & NA & NA & $\stackrel{+}{\ddagger}$ & NA & (9) \\
\hline B. microti sa1 & NA & NA & NA & $\ddagger$ & (10) \\
\hline B. microti $18 \mathrm{~S}$ rDNA & NA & NA & NA & \pm & (10) \\
\hline
\end{tabular}

seminested PCR and sequenced it to confirm $B$. burgdorferi sensu stricto-positive specimens. We followed real-time PCR and PepX amplification protocols published elsewhere (9). We amplified and sequenced a 667-nt fragment of the cytochrome oxidase subunit I (COI) gene using primers LCO1490 and HCO2198 (11) to confirm the tick species of positive specimens. The PCR mixture $(25 \mu \mathrm{L})$ contained forward and reverse primers at a final concentration of $0.4 \mu \mathrm{mol}$ and $5 \mu \mathrm{L}$ of DNA template. Thermocycling conditions followed protocols published elsewhere (11). COI and PepX amplicons were sequenced as described elsewhere (9).

\section{Results}

A total of $668 \mathrm{H}$. longicornis ticks (356 larvae, 166 nymphs, 146 adults) were collected from 4 counties in southeastern Pennsylvania (Figure). During the same period, $265 \mathrm{I}$. scapularis ticks (174 larvae, 78 nymphs, 13 adults) were collected from the same 4 counties. Of the subset of $H$. longicornis ticks tested by using real-time PCR $(\mathrm{n}=263), 1(0.4 \%)$ adult female collected from a county park in Bucks County on June 14, 2019 was positive for B. burgdorferi sensu stricto. A 570-nt segment of the PepX gene from this specimen was identical to B. burgdorferi sensu stricto reference sequences (GenBank accession nos. CP002312.1:657467-658036). The COI gene fragment from this tick showed $99.8 \%$ identity to an H. longicornis tick sequence in the GenBank database (accession no. JQ737090). No H. longicornis ticks were positive for B. miyamotoi, B. mayonii, or B. microti.

\section{Conclusions}

We document detection of the Lyme disease spirochete, $B$. burgdorferi sensu stricto, in invasive $H$. longicornis ticks. The overall infection rate of $0.4 \%$ was low. In comparison, B. burgdorferi sensu lato infection rates in I. scapularis ticks collected during the same surveillance period and in the same counties ranged from $16.7 \%$ to $57.1 \%$ (K.P. Price et al., unpub. data). This finding is consistent with recent findings that $H$. longicornis ticks are relatively averse to feeding on white-footed mice (Peromyscus leucopus), the primary reservoir of $B$. burgdorferi sensu stricto (12). Our findings support laboratory studies demonstrating that $H$. longicornis ticks can acquire B. burgdorferi sensu stricto while feeding on experimentally infected mice; however, those studies suggested that $H$. longicornis ticks are unlikely to contribute to transmission of B. burgdorferi sensu stricto because infection is lost during molting (13). However, refeeding and transmission of Lyme spirochetes by partially-fed ixodid ticks has been documented (14).

On the basis of microscopy, we estimated that $\approx 10 \%$ of the host-seeking H. longicornis ticks that we recovered were partially fed, suggesting the possibility that transmission could occur before the ticks molt. Of note, however, although we detected B. burgdorferi sensu stricto DNA in the tick, we have no evidence to suggest the spirochetes were viable. Unique ecologic traits of H. longicornis ticks (e.g., cold hardiness, parthenogenetic reproduction, host generality), which may enable the species' rapid establishment and high density (4), could confound efforts to determine the extent to which the tick may be involved in maintenance of $B$. burgdorferi sensu stricto in nature.

Continued monitoring to identify infested areas is essential, especially in densely populated regions (e.g., southeastern Pennsylvania). Despite limited documentation of $H$. longicornis ticks biting humans in the United States (15), findings presented here support continued use of personal protective measures. $H$. longicornis ticks are a vector of human pathogens in its native range; further investigation is needed to determine its potential public health significance in the United States. 

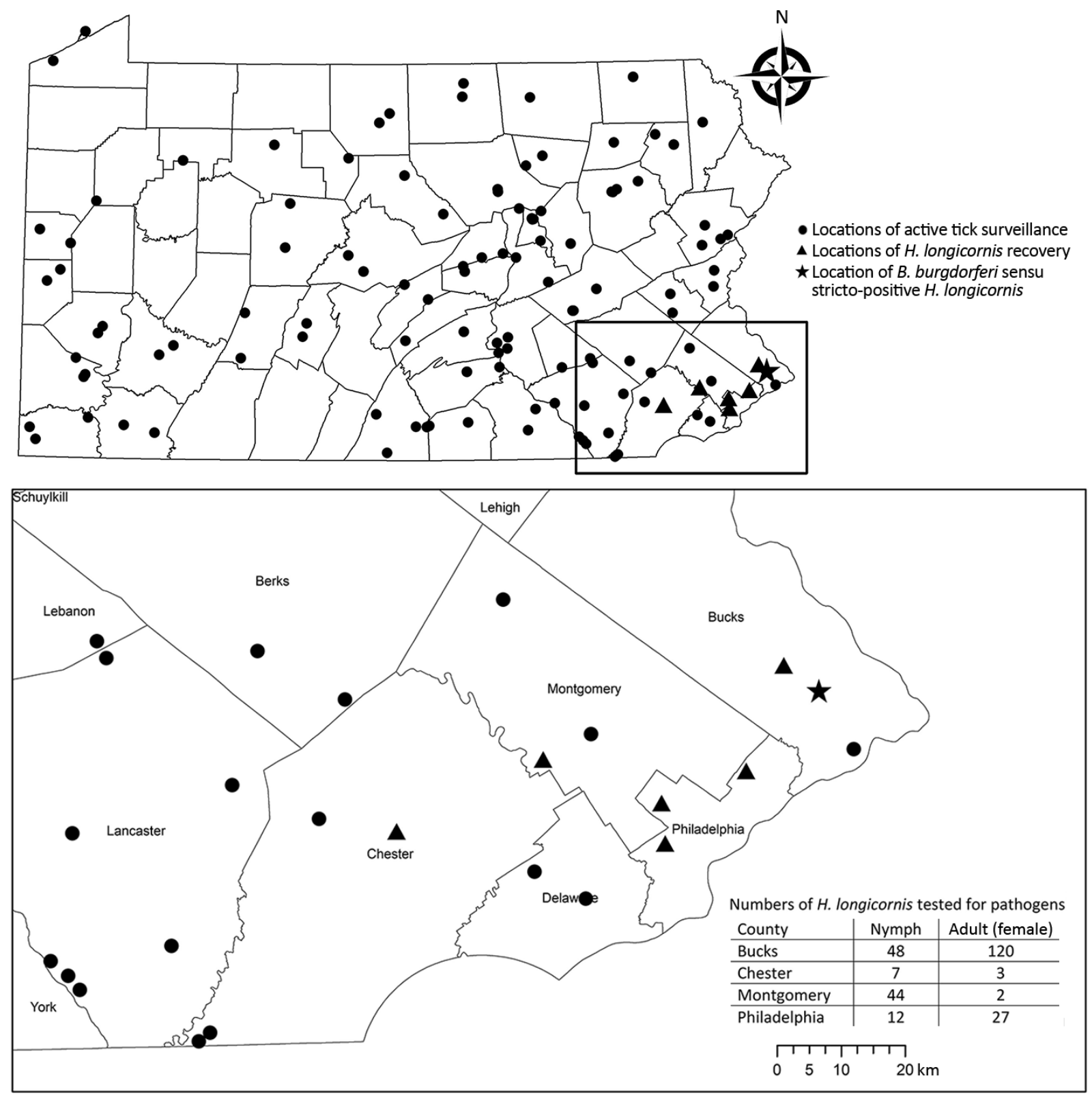

Figure. County map of Pennsylvania, USA, and the southeastern region (inset) showing locations of active tick surveillance, where Haemaphysalis longicornis ticks were recovered, and where Borrelia burgdorferi sensu stricto-positive H. longicornis ticks were found, May 1-September 6, 2019. Pennsylvania county map shows 38 counties sampled weekly and an additional 14 counties sampled opportunistically that yielded low tick recovery (Ixodes scapularis ticks only).

\section{Acknowledgments}

We thank field personnel for collection efforts, Rebecca

Eckert for helpful comments, and Pennsylvania

Department of Health, especially Leah Lind, for cooperation and support.

Funding for this study was provided, in part, by an epidemiology subgrant (\#4100082142) from the Pennsylvania Department of Health.

\section{About the Author}

Dr. Price is a microbiologist at the Pennsylvania

Department of Environmental Protection, Division of Vector Management, Harrisburg, PA. His research interests include vector ecology and vector-borne disease epidemiology.

\section{References}

1. Mead PS. Epidemiology of Lyme disease. Infect Dis Clin North Am. 2015;29:187-210. https://doi.org/10.1016/ j.idc. 2015.02 .010 
2. Hutchinson ML, Strohecker MD, Simmons TW, Kyle AD, Helwig MW. Prevalence rates of Borrelia burgdorferi (Spirochaetales: Spirochaetaceae), Anaplasma phagocytophilum (Rickettsiales: Anaplasmataceae), and Babesia microti (Piroplasmida: Babesiidae) in host-seeking Ixodes scapularis (Acari: Ixodidae) from Pennsylvania. J Med Entomol. 2015;52:693-8. https://doi.org/10.1093/jme/tjv037

3. Rainey T, Occi JL, Robbins RG, Egizi A. Discovery of Haemaphysalis longicornis (Ixodida: Ixodidae) parasitizing a sheep in New Jersey, United States. J Med Entomol. 2018;55:757-9. https://doi.org/10.1093/jme/tjy006

4. Price KJ, Witmier BJ, Eckert RA, Boyer CN, Helwig MW, Kyle AD. Distribution and density of Haemaphysalis longicornis (Acari: Ixodidae) on public lands in Pennsylvania, United States. J Med Entomol. 2020 Dec 25 [Epub ahead of print]. https://doi.org/10.1093/jme/tjaa274

5. Zhao L, Li J, Cui X, Jia N, Wei J, Xia L, et al. Distribution of Haemaphysalis longicornis and associated pathogens: analysis of pooled data from a China field survey and global published data. Lancet Planet Health. 2020;4:e320-9. https://doi.org/10.1016/S2542-5196(20)30145-5

6. Tufts DM, VanAcker MC, Fernandez MP, DeNicola A, Egizi A, Diuk-Wasser MA. Distribution, host-seeking phenology, and host and habitat associations of Haemaphysalis longicornis ticks, Staten Island, New York, USA. Emerg Infect Dis. 2019;25:792-6. https://doi.org/10.3201/eid2504.181541

7. Kingry LC, Anacker M, Pritt B, Bjork J, Respicio-Kingry L, Liu G, et al. Surveillance for and discovery of Borrelia species in US patients suspected of tickborne illness. Clin Infect Dis. 2018;66:1864-71. https://doi.org/10.1093/cid/cix1107

8. Zeidner NS, Schneider BS, Dolan MC, Piesman J. An analysis of spirochete load, strain, and pathology in a model of tick-transmitted Lyme borreliosis. Vector Borne Zoonotic Dis. 2001;1:35-44. https://doi.org/10.1089/153036601750137642

9. Graham CB, Maes SE, Hojgaard A, Fleshman AC, Sheldon SW, Eisen RJ. A molecular algorithm to detect and differentiate human pathogens infecting Ixodes scapularis and
Ixodes pacificus (Acari: Ixodidae). Ticks Tick Borne Dis. 2018;9:390-403. https:// doi.org/10.1016/ j.ttbdis.2017.12.005

10. Hojgaard A, Lukacik G, Piesman J. Detection of Borrelia burgdorferi, Anaplasma phagocytophilum and Babesia microti, with two different multiplex PCR assays. Ticks Tick Borne Dis. 2014;5:349-51. https:/ / doi.org/10.1016/ j.ttbdis.2013.12.001

11. Zhang R, Zhao A, Wang X, Zhang Z. Diversity of tick species on domestic animals in Shandong province, China, using DNA barcoding. Exp Appl Acarol. 2017;73:79-89. https:/ / doi.org/10.1007/s10493-017-0161-7

12. Ronai I, Tufts DM, Diuk-Wasser MA. Aversion of the invasive Asian longhorned tick to the white-footed mouse, the dominant reservoir of tick-borne pathogens in the U.S.A. Med Vet Entomol. 2020;34:369-73. https://doi.org/10.1111/ mve.12441

13. Breuner NE, Ford SL, Hojgaard A, Osikowicz LM, Parise CM, Rosales Rizzo MF, et al. Failure of the Asian longhorned tick, Haemaphysalis longicornis, to serve as an experimental vector of the Lyme disease spirochete, Borrelia burgdorferi sensu stricto. Ticks Tick Borne Dis. 2020;11:101311. https:/ / doi.org/ 10.1016/j.ttbdis.2019.101311

14. Nakao M, Sato Y. Refeeding activity of immature ticks of Ixodes persulcatus and transmission of Lyme disease spirochete by partially fed larvae. J Parasitol. 1996;82:669-72. https:/ / doi.org/10.2307/3283804

15. Wormser GP, McKenna D, Piedmonte N, Vinci V, Egizi AM, Backenson B, et al. First recognized human bite in the United States by the Asian longhorned tick, Haemaphysalis longicornis. Clin Infect Dis. 2020;70:314-6. https://doi.org/10.1093/cid/ciz449

Address for correspondence: Keith J. Price, Pennsylvania Department of Environmental Protection, 2575 Interstate Dr., Harrisburg, PA 17110, USA; email: keitprice@pa.gov 